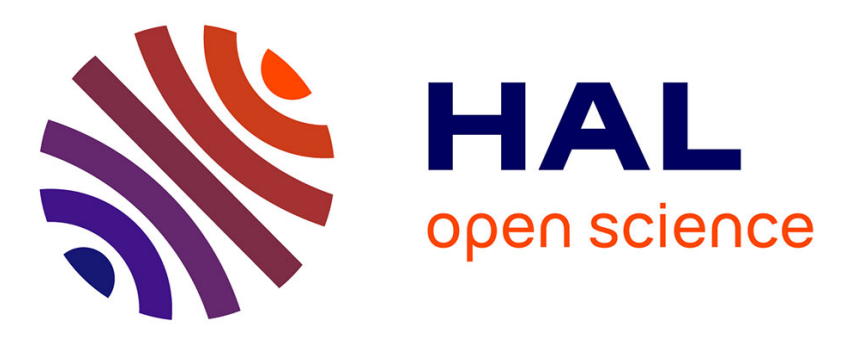

\title{
Molecular interactions governing the incorporation of cholecalciferol and retinyl-palmitate in mixed taurocholate-lipid micelles
}

Charles Desmarchelier, Véronique Rosilio, David Chapron, Ali Makky, Damien Preveraud, Estelle Devillard, Véronique Legrand-Defretin, Patrick Borel

\section{To cite this version:}

Charles Desmarchelier, Véronique Rosilio, David Chapron, Ali Makky, Damien Preveraud, et al.. Molecular interactions governing the incorporation of cholecalciferol and retinylpalmitate in mixed taurocholate-lipid micelles. Food Chemistry, 2018, 250, pp.221 - 229. 10.1016/j.foodchem.2018.01.063 . hal-01757864

\section{HAL Id: hal-01757864 \\ https://hal-amu.archives-ouvertes.fr/hal-01757864}

Submitted on 4 Apr 2018

HAL is a multi-disciplinary open access archive for the deposit and dissemination of scientific research documents, whether they are published or not. The documents may come from teaching and research institutions in France or abroad, or from public or private research centers.
L'archive ouverte pluridisciplinaire HAL, est destinée au dépôt et à la diffusion de documents scientifiques de niveau recherche, publiés ou non, émanant des établissements d'enseignement et de recherche français ou étrangers, des laboratoires publics ou privés. 
3 Charles Desmarchelier ${ }^{1}$, Véronique Rosilio ${ }^{2 *}$, David Chapron ${ }^{2}$, Ali Makky ${ }^{2}$, Damien P.

Molecular interactions governing the incorporation of cholecalciferol and retinylpalmitate in mixed taurocholate-lipid micelles
5

6

7

Prévéraud $^{3}$, Estelle Devillard $^{3}$, Véronique Legrand-Defretin $^{3}$, Patrick Borel $^{1}$ 列

${ }^{1}$ NORT, Aix-Marseille University, INRA, INSERM, 13005, Marseille, France.

${ }^{2}$ Institut Galien Paris Sud, UMR 8612, Univ Paris-Sud, CNRS, Université Paris-Saclay, 5 rue J.-B. Clément, 92290 Châtenay-Malabry, France.

${ }^{3}$ Adisseo France S.A.S., Center of Expertise and Research in Nutrition, 6 route Noire, 03600 Commentry, France.

11

2

*Corresponding author: Véronique Rosilio, Université Paris-Sud, UMR CNRS 8612, Université Paris-Saclay, 5 rue J-B. Clément, 92296 Châtenay-Malabry Cedex FRANCE. Tel : +33 1468354 18. E-Mail: veronique.rosilio@u-psud.fr

77

8

\footnotetext{
Abbreviations: NaTC (sodium taurocholate), RP (retinyl palmitate), $\mathrm{D}_{3}$ (cholecalciferol), cmc (critical micelle concentration), cac (critical aggregation concentration), LDP (lipid digestion products), PC (phosphatidylcholine), Lyso-PC (L- $\alpha$-lysophosphatidylcholine palmitoyl), POPC (2-oleoyl-1-palmitoyl-sn-glycero-3-phosphocholine).
}

24


Abstract

Cholecalciferol $\left(\mathrm{D}_{3}\right)$ and retinyl palmitate $(\mathrm{RP})$ are the two main fat-soluble vitamins found in

foods from animal origin. It is assumed that they are solubilized in mixed micelles prior to their uptake by intestinal cells, but only scarce data are available on the relative efficiency of this process and the molecular interactions that govern it. The extent of solubilization of $\mathrm{D}_{3}$ and $\mathrm{RP}$ in micelles composed of lipids and sodium taurocholate (NaTC) was determined. Then, the molecular interactions between components were analyzed by surface tension and surface pressure measurements. The mixture of lipids and NaTC allowed formation of micelles with higher molecular order, and at lower concentrations than pure NaTC molecules. $\mathrm{D}_{3}$ solubilization in the aqueous phase rich in mixed micelles was several times higher than that of RP. This was explained by interactions between NaTC or lipids and $\mathrm{D}_{3}$ thermodynamically more favorable than with $\mathrm{RP}$, and by $\mathrm{D}_{3}$ self-association.

Keywords: bioaccessibility; surface pressure; bile salt; compression isotherm; lipid monolayer; vitamin A; vitamin D; phospholipid.

\section{Introduction}

Retinyl esters and cholecalciferol $\left(\mathrm{D}_{3}\right)$ (Figure 1) are the two main fat-soluble vitamins found in foods of animal origin. There is a renewed interest in deciphering their absorption mechanisms because vitamin $\mathrm{A}$ and $\mathrm{D}$ deficiency is a public health concern in numerous countries, and it is thus of relevance to identify factors limiting their absorption to tackle this global issue. The fate of these vitamins in the human upper gastrointestinal tract during 
digestion is assumed to follow that of dietary lipids (Borel et al. 2015). This includes emulsification, solubilization in mixed micelles, diffusion across the unstirred water layer and uptake by the enterocyte via passive diffusion or apical membrane proteins (Reboul et al. 2011). Briefly, following consumption of vitamin-rich food sources, the food matrix starts to undergo degradation in the acidic environment of the stomach, which contains several enzymes, leading to a partial release of these lipophilic molecules and to their transfer to the lipid phase of the meal. Upon reaching the duodenum, the food matrix is further degraded by pancreatic secretions, promoting additional release from the food matrix, and both vitamins then transfer from oil-in-water emulsions to mixed micelles (and possibly other structures, such as vesicles, although not demonstrated yet). As it is assumed that only free retinol can be taken up by enterocytes, retinyl esters are hydrolyzed by pancreatic enzymes, namely pancreatic lipase, pancreatic lipase-related protein 2 and cholesterol ester hydrolase (Desmarchelier et al. 2013). Bioaccessible vitamins are then taken up by enterocytes via simple passive diffusion or facilitated diffusion mediated by apical membrane proteins (Desmarchelier et al. 2017). The apical membrane protein(s) involved in retinol uptake by enterocytes is(are) yet to be identified but in the case of $\mathrm{D}_{3}$, three proteins have been shown to facilitate its uptake: NPC1L1 (NPC1 like intracellular cholesterol transporter 1), SR-BI (scavenger receptor class B member 1) and CD36 (Cluster of differentiation 36) (Reboul \& Borel 2011). Both vitamins then transfer across the enterocyte towards the basolateral side. The transfer of vitamin A is mediated, at least partly, by the cellular retinol-binding protein, type II (CRBPII), while that of vitamin D is carried out by unknown mechanisms. Additionally, a fraction of retinol is re-esterified by several enzymes (Borel \& Desmarchelier 2017). Vitamin A and D are then incorporated in chylomicrons in the Golgi apparatus before secretion in the lymph.

The solubilization of vitamins A and D in mixed micelles, also called micellarization or micellization, is considered as a key step for their bioavailability because it is assumed that the 
non-negligible fraction of fat-soluble vitamin that is not micellarized is not absorbed (Desmarchelier et al. 2013). Mixed micelles are mainly made of a mixture of bile salts, phospholipids and lysophospholipids, cholesterol, fatty acids and monoglycerides (Hernell et al. 1990). These compounds may form various self-assembled structures, e.g., spherical, cylindrical or disk-shaped micelles (Walter et al. 1991, Leng et al. 2003) or vesicles, depending on their concentration, the bile salt/phospholipid ratio (Walter et al. 1991), the phospholipid concentration, but also the ionic strength, $\mathrm{pH}$ and temperature of the aqueous medium (Madency \& Egelhaaf 2010; Salentinig et al. 2010; Cheng et al. 2014). Fat-soluble micronutrients display large variations with regards to their solubility in mixed micelles (Sy et al. 2012; Gleize et al. 2016) and several factors are assumed to account for these differences (Desmarchelier \& Borel 2017, for review).

The mixed micelle lipid composition has been shown to significantly affect vitamin absorption. For example, the substitution of lysophospholipids by phospholipids diminished the lymphatic absorption of vitamin $\mathrm{E}$ in rats (Koo et al. 2001). In rat perfused intestine, the addition of fatty acids of varying chain length and saturation degree, i.e. butyric, octanoic, oleic and linoleic acid, resulted in a decrease in the rate of $\mathrm{D}_{3}$ absorption (Hollander et al. 1978). The effect was more pronounced in the ileal part of the small intestine following the addition of oleic and linoleic acid. It was suggested that unlike short- and medium-chain fatty acids, which are not incorporated into micelles, long-chain fatty acids hinder vitamin D absorption by causing enlargement of micelle size, thereby slowing their diffusion towards the enterocyte. Moreover, the possibility that $\mathrm{D}_{3}$ could form self-aggregates in water (Meredith et al. 1984), although not clearly demonstrated, has led to question the need of mixed micelles for its solubilization in the aqueous environment of the intestinal tract lumen (Rautureau and Rambaud 1981; Maislos and Shany 1987). 

phase rich in mixed micelles that exists in the upper intestinal lumen during digestion, and to dissect, by surface tension and surface pressure measurements, the molecular interactions existing between these vitamins and the mixed micelle components that explain the different solubility of $\mathrm{D}_{3}$ and $\mathrm{RP}$ in mixed micelles.

\section{Materials and methods}

\subsection{Chemicals}

2-oleoyl-1-palmitoyl-sn-glycero-3-phosphocholine (POPC) (phosphatidylcholine, $\geq 99 \% ; \quad$ Mw $\quad 760.08 \quad \mathrm{~g} / \mathrm{mol}$ ), $\quad$ 1-palmitoyl-sn-glycero-3-phosphocholine $\quad$ (Lyso-PC) (lysophosphatidylcholine, $\geq 99 \%$; Mw $495.63 \mathrm{~g} / \mathrm{mol}$ ), free cholesterol ( $\geq 99 \%$; Mw 386.65 g/mol), oleic acid (reagent grade, $\geq 99 \%$; Mw $282.46 \mathrm{~g} / \mathrm{mol}$ ), 1-monooleoyl-rac-glycerol (monoolein, C18:1,-cis-9, Mw $356.54 \mathrm{~g} / \mathrm{mol}$ ), taurocholic acid sodium salt hydrate (NaTC) ( $\geq 95 \%$; Mw $537.68 \mathrm{~g} / \mathrm{mol}$ ) ), cholecalciferol (>98\%; Mw $384.64 \mathrm{~g} / \mathrm{mol}$; melting point $84.5^{\circ} \mathrm{C}$; solubility in water: $10^{-4}-10^{-5} \mathrm{mg} / \mathrm{mL}$; $\left.\log \mathrm{P} 7.5\right)$ and retinyl palmitate $(>93.5 \%$; Mw 524.86 $\mathrm{g} / \mathrm{mol}$; melting point $28.5^{\circ} \mathrm{C}$; $\left.\log \mathrm{P} 13.6\right)$ were purchased from Sigma-Aldrich (Saint-QuentinFallavier, France). Chloroform and methanol (99\% pure) were analytical grade reagents from Merck (Germany). Ethanol (99.9\%), n-hexane, chloroform, acetonitrile, dichloromethane and methanol were HPLC grade reagents from Carlo Erba Reagent (Peypin, France). Ultrapure water was produced by a Milli-Q ${ }^{\circledR}$ Direct 8 Water Purification System (Millipore, Molsheim, France). Prior to all surface tension, and surface pressure experiments, all glassware was soaked for an hour in a freshly prepared hot TFD4 (Franklab, Guyancourt, France) detergent solution $(15 \% \mathrm{v} / \mathrm{v})$, and then thoroughly rinsed with ultrapure water. Physico-chemical properties of $\mathrm{D}_{3}$ and RP were retrieved from PubChem (https://pubchem.ncbi.nlm.nih.gov/). 


\subsection{Micelle formation}

The micellar mixture contained $0.3 \mathrm{mM}$ monoolein, $0.5 \mathrm{mM}$ oleic acid, $0.04 \mathrm{mM}$ POPC, $0.1 \mathrm{mM}$ cholesterol, $0.16 \mathrm{mM}$ Lyso-PC, and $5 \mathrm{mM} \mathrm{NaTC}$ (Reboul et al. 2005). Total component concentration was thus $6.1 \mathrm{mM}$, with NaTC amounting to $82 \mathrm{~mol} \%$. Two vitamins were studied: crystalline $\mathrm{D}_{3}$ and $\mathrm{RP}$.

Mixed micelles were formed according to the protocol described by Desmarchelier et al. (2013). Lipid digestion products (LDP) (monoolein, oleic acid, POPC, cholesterol and Lyso$\mathrm{PC}$, total concentration $1.1 \mathrm{mM})$ dissolved in chloroform/methanol $(2: 1, \mathrm{v} / \mathrm{v})$, and $\mathrm{D}_{3}$ or $\mathrm{RP}$ dissolved in ethanol were transferred to a glass tube and the solvent mixture was carefully evaporated under nitrogen. The dried residue was dispersed in Tris buffer (Tris-HCl $1 \mathrm{mM}$, $\mathrm{CaCl}_{2}$ 5mM, $\mathrm{NaCl} 100 \mathrm{mM}, \mathrm{pH} 6.0$ ) containing $5 \mathrm{mM}$ taurocholate, and incubated at $37^{\circ} \mathrm{C}$ for $30 \mathrm{~min}$. The solution was then vigorously mixed by sonication at $25 \mathrm{~W}$ (Branson $250 \mathrm{~W}$ sonifier; Danbury, CT, U.S.A.) for $2 \mathrm{~min}$, and incubated at $37^{\circ} \mathrm{C}$ for 1 hour. To determine the amount of vitamin solubilized in structures allowing their subsequent absorption by enterocytes (bioaccessible fraction), i.e. micelles and possibly small lipid vesicles, whose size is smaller than that of mucus pores (Cone 2009), the solutions were filtered through cellulose ester membranes $(0.22 \mu \mathrm{m})$ (Millipore), according to Tyssandier et al. 2003. The resulting optically clear solution was stored at $-20{ }^{\circ} \mathrm{C}$ until vitamin extraction and HPLC analysis. $\mathrm{D}_{3}$ and RP concentrations were measured by HPLC before and after filtration. For surface tension measurements and cryoTEM experiments, the mixed micelle systems were not filtered.

\subsection{Self-micellarization of $\mathrm{D}_{3}$}

Molecular assemblies of $\mathrm{D}_{3}$ were prepared in Tris buffer using the same protocol as for mixed micelles. $\mathrm{D}_{3}$ was dissolved into the solvent mixture and after evaporation, the dry film was hydrated for $30 \mathrm{~min}$ at $37^{\circ} \mathrm{C}$ with taurocholate-free buffer. The suspension was then 
sonicated. All $\mathrm{D}_{3}$ concentrations reported in the surface tension measurements were obtained from independent micellarization experiments - not from the dilution of one concentrated $\mathrm{D}_{3}$ solution.

\subsection{Surface tension measurements}

Mixed micelle solutions were prepared as described above, at concentrations ranging from $5.5 \mathrm{nM}$ to $55 \mathrm{mM}$, with the same proportion of components as previously mentioned. The surface tension of LDP mixtures hydrated with a taurocholate-free buffer, and that of pure taurocholate solutions were also measured at various concentrations. The solutions were poured into glass cuvettes. The aqueous surface was cleaned by suction, and the solutions were left at rest under saturated vapor pressure for 24 hours before measurements. For penetration studies, glass cuvettes with a side arm were used, allowing injection of NaTC beneath a spread LDP or vitamin monolayer. Surface tension measurements were performed by the Wilhelmy plate method, using a thermostated automatic digital tensiometer (K10 Krüss, Germany). The surface tension $\gamma$ was recorded continuously as a function of time until equilibrium was reached. All experiments were performed at $25 \pm 1^{\circ} \mathrm{C}$ under saturated vapor pressure to maintain a constant level of liquid. The reported values are mean of three measurements. The experimental uncertainty was estimated to be $0.2 \mathrm{mN} / \mathrm{m}$. Surface pressure $(\pi)$ values were deduced from the relationship $\pi=\gamma_{0}-\gamma$, with $\gamma_{0}$ the surface tension of the subphase and $\gamma$ the surface tension in the presence of a film.

\subsection{Surface pressure measurements}

Surface pressure-area $\pi$-A isotherms of the LDP and LDP-vitamin mixtures were obtained using a thermostated Langmuir film trough $\left(775.75 \mathrm{~cm}^{2}\right.$, Biolin Scientific, Finland) enclosed into a Plexiglas box (Essaid et al. 2016). Solutions of lipids in a chloroform/methanol 
$(9: 1, \mathrm{v} / \mathrm{v})$ mixture were spread onto a clean buffer subphase. Monolayers were left at rest for 20 minutes to allow complete evaporation of the solvents. They were then compressed at low speed $\left(6.5 \AA^{2} \cdot\right.$ molecule $\left.{ }^{-1} \cdot \mathrm{min}^{-1}\right)$ to minimize the occurrence of metastable phases. The experimental uncertainty was estimated to be $0.1 \mathrm{mN} / \mathrm{m}$. All experiments were run at $25 \pm 1{ }^{\circ} \mathrm{C}$. Mean isotherms were deduced from at least three compression isotherms. The surface compressional moduli $\mathrm{K}$ of monolayers were calculated using Eq. 1:

$$
K=-A\left(\frac{d \pi}{d A}\right)_{T}(\text { Eq. } 1)
$$

Excess free energies of mixing were calculated according to Eq. 2:

$$
\Delta G^{E X C}=\int_{0}^{\pi}\left(X_{L} A_{L}+X_{V I T} A_{V I T}\right) d \pi(\text { Eq. 2) }
$$

with $\mathrm{X}_{\mathrm{L}}$ and $\mathrm{A}_{\mathrm{L}}$ the molar fraction and molecular area of lipid molecules, and $\mathrm{X}_{\mathrm{VIT}}$ and $\mathrm{A}_{\mathrm{VIT}}$ the molar fraction and molecular area of vitamin molecules, respectively (Ambike et al. 2011).

\subsection{Cryo-TEM analysis}

A drop $(5 \mu \mathrm{L})$ of LDP-NaTC micellar solution $(15 \mathrm{mM})$, LDP-NaTC-D $_{3}$ (3:1 molar ratio) or pure $\mathrm{D}_{3}$ "micellar suspension" ( $5 \mathrm{mM}$, theoretical concentration) was deposited onto a perforated carbon-coated, copper grid (TedPella, Inc); the excess of liquid was blotted with a filter paper. The grid was immediately plunged into a liquid ethane bath cooled with liquid nitrogen $\left(180^{\circ} \mathrm{C}\right)$ and then mounted on a cryo holder (Da Cunha et al. 2016). Transmission electron measurements (TEM) measurements were performed just after grid preparation using a JEOL 2200FS (JEOL USA, Inc., Peabody, MA, U.S.A.) working under an acceleration voltage of $200 \mathrm{kV}$ (Institut Curie). Electron micrographs were recorded by a CCD camera (Gatan, Evry, France). 
2.7.1. Vitamin extraction.

$\mathrm{D}_{3}$ and RP were extracted from $500 \mu \mathrm{L}$ aqueous samples using the following method

(Desmarchelier et al. 2013): retinyl acetate was used as an internal standard and was added to the samples in $500 \mu \mathrm{L}$ ethanol. The mixture was extracted twice with two volumes of hexane. The hexane phases obtained after centrifugation $\left(1200 \times \mathrm{g}, 10 \mathrm{~min}, 10^{\circ} \mathrm{C}\right)$ were evaporated to dryness under nitrogen, and the dried extract was dissolved in $200 \mu \mathrm{L}$ of acetonitrile/dichloromethane/methanol (70:20:10, v/v/v). A volume of $150 \mu \mathrm{L}$ was used for HPLC analysis. Extraction efficiency was between 75 and 100\%. Sample whose extraction efficiency was below $75 \%$ were re-extracted or taken out from the analysis.

2.7.2. Vitamin HPLC analysis.

$\mathrm{D}_{3}$, and RP and retinyl acetate were separated using a $250 \times 4.6-\mathrm{nm}$ RP C18, 5- $\mu \mathrm{m}$ Zorbax Eclipse XDB column (Agilent Technologies, Les Ulis, France) and a guard column. The mobile phase was a mixture of acetonitrile/dichloromethane/methanol $(70: 20: 10, \mathrm{v} / \mathrm{v} / \mathrm{v})$. Flow rate was $1.8 \mathrm{~mL} / \mathrm{min}$ and the column was kept at a constant temperature $\left(35^{\circ} \mathrm{C}\right)$. The HPLC system comprised a Dionex separation module (P680 HPLC pump and ASI-100 automated sample injector, Dionex, Aix-en-Provence, France). $\mathrm{D}_{3}$ was detected at $265 \mathrm{~nm}$ while retinyl esters were detected at $325 \mathrm{~nm}$ and were identified by retention time compared with pure $(>95 \%)$ standards. Quantification was performed using Chromeleon software (version 6.50, SP4 Build 1000) comparing the peak area with standard reference curves. All solvents used were HPLC grade. 
Results are expressed as means \pm standard deviation. Statistical analyses were

performed using Statview software version 5.0 (SAS Institute, Cary, NC, U.S.A.). Means were compared by the non-parametric Kruskal-Wallis test, followed by Mann-Whitney U test as a post hoc test for pairwise comparisons, when the mean difference using the Kruskal-Wallis test was found to be significant $(P<0.05)$. For all tests, the bilateral alpha risk was $\alpha=0.05$.

\section{Results}

\subsection{Solubilization of $D_{3}$ and $R P$ in aqueous solutions rich in mixed micelles}

$\mathrm{D}_{3}$ and RP at various concentrations were mixed with micelle components (LDP-NaTC).

$\mathrm{D}_{3}$ and RP concentrations were measured by HPLC before and after filtration of aggregates with a diameter smaller than $0.22 \mu \mathrm{m}$ (Figure 2). $\mathrm{D}_{3}$ and RP solubilization in the solution that contained mixed micelle solution followed different curves: $\mathrm{D}_{3}$ solubilization was linear $\left(\mathrm{R}^{2}=0.98\right.$, regression slope $\left.=0.71\right)$ and significantly higher than that of $\mathrm{RP}$, which reached a plateau with a maximum concentration around $125 \mu \mathrm{M}$.

The morphology of the LDP-NaTC and LDP-NaTC-D 3 samples before filtration was analyzed by cryoTEM. In Figure 3, micelles are too small to be distinguished from ice. At high LDP-NaTC concentration $(15 \mathrm{mM})$ small and large unilamellar vesicles $(a)$, nano-fibers $(b)$ and aggregates $(c)$ are observed (Figure 3A). Both nano-fibers and aggregates seem to emerge from the vesicles. In the presence of $\mathrm{D}_{3}$ at low micelle and D3 concentration (5 mM LDP-NaTC + $1.7 \mathrm{mM} \mathrm{D}_{3}$ ) (Figures 3B and 3C), the morphology of the nano-assemblies is greatly modified. Vesicles are smaller and deformed, with irregular and more angular shapes $\left(a^{\prime}\right)$. There are also more abundant. A difference in contrast in the bilayers is observed, which would account for leaflets with asymmetric composition. Some of them coalesce into larger structures, extending along the walls of the grid $(d)$. Fragments and sheets are also observed (figure 3B). They exhibit 
irregular contour and unidentified membrane organization. The bilayer structure is not clearly observable. New organized assemblies appear, such as disk-like nano-assemblies $(e)$ and emulsion-like droplets $(f)$. At higher concentration (15 $\mathrm{mM}$ LDP-NaTC $+5 \mathrm{mM} \mathrm{D}$ in figure 3D), the emulsion-like droplets and vesicles with unidentified membrane structure $(g)$ are enlarged. They coexist with small deformed vesicules.

\subsection{Compression properties of LDP components, the LDP mixture and the vitamins}

To better understand the mechanism of $\mathrm{D}_{3}$ and RP interaction with LDP-NaTC micelles, we focused on the interfacial behavior of the various components of the system. We first determined the interfacial behavior of the LDP components and their mixture in proportions similar to those in the micellar solution, by surface pressure measurements. The $\pi$-A isotherms are plotted in Figure 4A. Based on the calculated compressibility modulus values, the lipid monolayers can be classified into poorly organized $(\mathrm{K}<100 \mathrm{mN} / \mathrm{m}$, for lyso-PC, monoolein, and oleic acid), liquid condensed $(100<\mathrm{K}<250 \mathrm{mN} / \mathrm{m}$, for POPC and the LDP mixture) and highly rigid monolayers ( $\mathrm{K}>250 \mathrm{mN} / \mathrm{m}$, for cholesterol) (Davies \& Rideal 1963).

The interfacial behavior of the two studied vitamins is illustrated in Figure 4B. $\mathrm{D}_{3}$ shows a similar compression profile to that of the LDP mixture, with comparable surface area and surface pressure at collapse $\left(\mathrm{A}_{\mathrm{c}}=35 \AA^{2}, \pi_{\mathrm{c}}=38 \mathrm{mN} / \mathrm{m}\right)$ but a much higher rigidity, as inferred from the comparison of their maximal $\mathrm{K}$ values $\left(187.4 \mathrm{mN} / \mathrm{m}\right.$ and $115.4 \mathrm{mN} / \mathrm{m}$ for $\mathrm{D}_{3}$ and $\mathrm{LDP}$, respectively). RP exhibits much larger surface areas and lower surface pressures than $\mathrm{D}_{3}$. The collapse of its monolayer is not clearly identified from the isotherms, and is estimated to occur at $\pi_{\mathrm{c}}=16.2 \mathrm{mN} / \mathrm{m}\left(\mathrm{A}_{\mathrm{c}}=56.0 \AA^{2}\right)$, as deduced from the slope change in the $\pi$-A plot.

\subsection{Self-assembling properties of $D_{3}$ in an aqueous solution}


Since $\mathrm{D}_{3}$ showed an interfacial behavior similar to that of the lipid mixture, and since it

could be solubilized at very high concentrations in an aqueous phase rich in mixed micelles (as shown in Figure 2), its self-assembling properties were more specifically investigated. Dried $\mathrm{D}_{3}$ films were hydrated with the sodium taurocholate free-buffer. Surface tension measurements at various $\mathrm{D}_{3}$ concentrations revealed that the vitamin could adsorb at the air/solution interface, and significantly lower the surface tension of the buffer to $\gamma_{\mathrm{cmc}}=30.6 \mathrm{mN} / \mathrm{m}$. A critical micellar concentration $(\mathrm{cmc}=0.45 \mu \mathrm{M})$ could be deduced from the $\gamma$ - $\log \mathrm{C}$ relationships and HPLC assays. Concentrated samples $\mathrm{D}_{3}$ samples were analyzed by cryo-TEM (Figure 3E and 3F). Different $\mathrm{D}_{3}$ self-assemblies were observed, including circular nano-assemblies $(h)$ coexisting with nano-fibers $(i)$, and large aggregates $(j)$ with unidentified structure. The analysis in depth of the circular nano-assemblies allowed to conclude that they were disk-like nano-assemblies, rather than nanoparticles.

\subsection{Interaction of LDP with NaTC}

To better understand how the two studied vitamins interacted with the mixed micelles, we compared the interfacial behaviors of the pure NaTC solutions, LDP mixtures hydrated by NaTC-free buffer, and LDP mixtures hydrated by the NaTC buffered solutions (full mixed micelle composition). The LDP mixture composition was maintained constant, while its concentration in the aqueous medium was increased. The concentration of NaTC in the aqueous phase was also calculated so that the relative proportion of the various components (LDP and NaTC) remained unchanged in all experiments. From the results plotted in Figure 5A, the critical micellar concentration (cmc) of the LDP-NaTC mixture was $0.122 \mathrm{mM}\left(\gamma_{\mathrm{cmc}}=29.0\right.$ $\mathrm{mN} / \mathrm{m}$ ), a concentration 50.8 times lower than the concentration used for vitamin solubilization. The $\mathrm{cmc}$ values for the LDP mixture and the pure NaTC solutions were $0.025 \mathrm{mM}\left(\gamma_{\mathrm{cmc}}=24.0\right.$ $\mathrm{mN} / \mathrm{m})$, and $1.5 \mathrm{mM}\left(\gamma_{\mathrm{cmc}}=45.3 \mathrm{mN} / \mathrm{m}\right)$, respectively. 
Experiments modeling the insertion of NaTC into the LDP film during rehydration by the buffer suggested that only few NaTC molecules could penetrate in the condensed LDP film (initial surface pressure: $\pi_{\mathrm{i}}=28 \mathrm{mN} / \mathrm{m}$ ) and that the LDP-NaTC mixed film was not stable, as shown by the decrease in surface pressure over time (Figure 5B).

\subsection{Interaction of $D_{3}$ and $R P$ with NaTC}

The surface tension of the mixed NaTC-LDP micelle solutions was only barely affected by the addition of 0.1 or $1 \mathrm{mM} \mathrm{D}_{3}$ or RP: the surface tension values increased by no more than $2.8 \mathrm{mN} / \mathrm{m}$. Conversely, both vitamins strongly affected the interfacial behavior of the NaTC micellar solution, as inferred from the significant surface tension lowering observed (- 7.0 and - $8.1 \mathrm{mN} / \mathrm{m}$ for $\mathrm{RP}$ and $\mathrm{D}_{3}$, respectively).

\subsection{Interaction of $D_{3}$ and $R P$ with lipid digestion products}

The interaction between the vitamins and LDP molecules following their insertion into LDP micelles was modeled by compression of $\mathrm{LDP} / \mathrm{D}_{3}$ and LDP/RP mixtures at a 7:3 molar ratio. This ratio was chosen arbitrarily, to model a system in which LDP was in excess. The $\pi-$ $\mathrm{A}$ isotherms are presented in Figures $6 \mathrm{~A}$ and $6 \mathrm{~B}$. They show that both vitamins modified the isotherm profile of the lipid mixture, however, not in the same way. In the LDP/D 3 mixture, the surface pressure and molecular area at collapse were controlled by LDP. For LDP/RP, despite the high content in LDP, the interfacial behavior was clearly controlled by RP. From the isotherms in Figures 6A and 6B, compressibility moduli and excess free energies of mixing were calculated and compared (Figures $6 \mathrm{C}$, and $6 \mathrm{D}$ ). $\mathrm{D}_{3}$ increased the rigidity of LDP monolayers, whereas RP disorganized them. The negative $\Delta \mathrm{G}^{E X C}$ values calculated for the LDP$D_{3}$ monolayers at all surface pressures account for the good mixing properties of $D_{3}$ and the 
lipids in all molecular packing conditions. Conversely for $\mathrm{RP}$, the positive and increasing $\Delta \mathrm{G}^{E X C}$ values with the surface pressure demonstrate that its interaction with the lipids was unfavorable.

\section{Discussion}

The objective of this study was to compare the solubility of $\mathrm{RP}$ and $\mathrm{D}_{3}$ in aqueous solutions containing mixed micelles, and to decipher the molecular interactions that explain their different extent of solubilization. Our first experiment revealed that the two vitamins exhibit very different solubilities in an aqueous medium rich in mixed micelles. Furthermore, the solubility of $\mathrm{D}_{3}$ was so high that we did not observe any limit, even when $\mathrm{D}_{3}$ was introduced at a concentration $>1 \mathrm{mM}$ in the aqueous medium. To our knowledge, this is the first time that such a difference is reported. Cryo-TEM pictures showed that $\mathrm{D}_{3}$ dramatically altered the organization of the various components of the mixed micelles. The spherical vesicles were deformed with angular shapes. The nano-fibers initiating from the vesicles were no longer observed. Large irregular in shape vesicle and sheets, disk-like nano-assemblies and emulsionlike droplets appeared in LDP-NaTC-D 3 mixtures, only. The existence of so many different assemblies would account for a different interaction of $\mathrm{D}_{3}$ with the various components of mixed micelles, and for a reorganization of the components. $\mathrm{D}_{3}$ could insert in the bilayer of vesicles and deform them, but also form emulsion-like droplets with fatty acids and monoglyceride. It is noteworthy that these emulsion-like droplets were not observed in pure $\mathrm{D}_{3}$ samples, nor mixed micelles. Since previous studies have shown that both bile salts and some mixed micelle lipids, e.g. fatty acids and phospholipids, can modulate the solubility of fatsoluble vitamins in these vehicles (Yang \& McClements 2013), we decided to study the 
interactions of these two vitamins with either bile salts or micelle lipids to assess the specific role of each component on vitamin solubility in mixed micelles.

The characteristics of pure POPC, Lyso-PC, monoolein, and cholesterol isotherms were in agreement with values published in the literature (Pezron et al. 1991; Flasinsky et al. 2014; Huynh et al. 2014). For oleic acid, the surface pressure at collapse was higher $\left(\pi_{\mathrm{c}}=37 \mathrm{mN} / \mathrm{m}\right)$ and the corresponding molecular area $\left(A_{c}=26 \AA^{2}\right)$ smaller than those previously published (Tomoaia-Cotisel et al. 1987), likely due to the $\mathrm{pH}$ of the buffer solution (pH 6) and the presence of calcium.

The interfacial properties of $\mathrm{D}_{3}$ were close to those deduced from the isotherm published by Meredith et al. (1984) for a $\mathrm{D}_{3}$ monolayer spread from a benzene solution onto a pure water subphase. The molecular areas at collapse are almost identical in the two studies (about $36 \AA^{2}$ ), but the surface pressures differ $(30 \mathrm{mN} / \mathrm{m}$ in Meredith and coworkers' study, and $38 \mathrm{mN} / \mathrm{m}$ in ours). Compressibility modulus values show that $\mathrm{D}_{3}$ molecules form monolayers with higher molecular order than the LDP mixture, which suggests that they might easily insert into LDP domains.

As could be expected from its chemical structure, RP exhibited a completely different interfacial behavior compared to $\mathrm{D}_{3}$ and the LDP, even to lyso-PC which formed the most expanded monolayers of the series, and displayed the lowest collapse surface pressure. The anomalous isotherm profile of lyso-PC has been attributed to monolayer instability and progressive solubilization of molecules into the aqueous phase (Heffner et al. 2013). The molecular areas and surface pressures for RP have been compared to those measured by Asai and Watanabe (2000) for RP monolayers spread from benzene solutions at $25^{\circ} \mathrm{C}$ onto a water subphase. Their values are much lower than ours, accounting for even more poorly organized monolayers. The low collapse surface pressure could correspond to molecules partially lying onto the aqueous surface, possibly forming multilayers above $16 \mathrm{mN} / \mathrm{m}$ as inferred from the 
continuous increase in surface pressure above the change in slope of the isotherm. The maximal compressibility modulus confirms the poor monolayer order. The significant differences in RP surface pressure and surface area compared to the LDP mixture might compromise its insertion and stability into LDP domains.

The dogma in nutrition is that fat-soluble vitamins need to be solubilized in bile salt micelles to be transported to the enterocyte and then absorbed. It is also well known that although NaTC primary micelles can be formed at 2-3 $\mathrm{mM}$ with a small aggregation number, concentrations as high as $10-12 \mathrm{mM}$ are usually necessary for efficient lipid solubilization in the intestine (Baskin \& Frost 2008). Due to their chemical structure bile salts have a facial arrangement of polar and non-polar domains (Madency \& Egelhaaf 2010). Their selfassembling (dimers, multimers, micelles) is a complex process involving hydrophobic interaction and cooperative hydrogen bonding, highly dependent on the medium conditions, and that is not completely elucidated. The cmc value for sodium taurocholate in the studied buffer was particularly low compared to some of those reported in the literature for NaTC in water or sodium chloride solutions (3-12 mM) (Kratohvil et al. 1983; Meyerhoffer \& Mcgown 1990; Madenci \& Egelhaaf 2010). At concentrations as high as 10-12 mM, NaTC molecules form elongated cylindrical "secondary" micelles (Madenci \& Egelhaaf 2010; Bottari et al. 1999). The cryoTEM analysis did not allow to distinguish micelles from the ice. In our solubilization experiment, the concentration of NaTC did not exceed $5 \mathrm{mM}$. Nevertheless, the micelles proved to be very efficient with regards to vitamin solubilization.

When bile salts and lipids are simultaneously present in the same environment, they form mixed micelles (Hernell et al. 1990). Bile salts solubilize phospholipid vesicles and transform into cylindrical micelles (Cheng et al. 2014). Walter et al (1991) suggested that sodium cholate cylindrical micelles evolved from the edge of lecithin bilayer sheets. Most published studies were performed at high phospholipid/bile salt ratio. In our system, the 
concentration of the phospholipids was very low compared to that of NaTC. We observed however the presence of vesicles, and nano-fiber structures emerging from them. In their cryoTEM analysis, Fatouros et al. (2009) compared bile salt/phospholipid mixtures to bile salt/phospholipid/fatty acid/monoglyceride ones at concentrations closer to ours. They observed only micelles in bile salt/phospholipid mixtures. However, in the presence of oleic acid and monoolein, vesicles and bilayer sheets were formed. This would account for a reorganization of the lipids and bile salts in the presence of the fatty acid and the monoglyceride. We therefore decided to study the interactions between bile salts and LDP. The results obtained show that the surface tension, the effective surface tension lowering concentration, and $\mathrm{cmc}$ values were very much influenced by LDP. The almost parallel slopes of Gibbs adsorption isotherms for pure NaTC and mixed NaTC-LDP suggest that LDP molecules inserted into NaTC domains, rather than the opposite. This was confirmed by penetration studies, which showed that NaTC $(0.1 \mathrm{mM})$ could hardly penetrate in a compact LDP film. So, during lipid hydration, LDP molecules could insert into NaTC domains. The presence of LDP molecules improved NaTC micellarization.

After having determined the interfacial properties of each micelle component and measured the interactions between NaTC and LDP, we assessed the ability of $\mathrm{D}_{3}$ and RP to solubilize in either NaTC or NaTC-LDP micelles. Surface tension values clearly show that both vitamins could insert in between NaTC molecules adsorbed at the interface, and affected the surface tension in the same way. The interfacial behavior of the molecules being representative of their behavior in the bulk, it is reasonable to think that both $\mathrm{D}_{3}$ and RP can be solubilized into pure NaTC micelles. For the mixed NaTC-LDP micelles, the change in surface tension was too limited to allow conclusions, but the solubilization experiments clearly indicated that neither vitamin was solubilized to the same extent. 

why the LDP-NaTC mixed micelles solubilize $\mathrm{D}_{3}$ better than RP. Therefore, we studied the interfacial behavior of the LDP mixture in the presence of each vitamin, to determine the extent of their interaction with the lipids. The results obtained showed that $\mathrm{D}_{3}$ penetrated in LDP domains and remained in the lipid monolayer throughout compression. At large molecular areas, the $\pi-\mathrm{A}$ isotherm profile of the mixture followed that of the LDP isotherm with a slight condensation due to the presence of $\mathrm{D}_{3}$ molecules. Above $10 \mathrm{mN} / \mathrm{m}$, an enlargement of the molecular area at collapse and a change in the slope of the mixed monolayer was observed. However, the surface pressure at collapse was not modified, and the shape of the isotherm accounted for the insertion of $\mathrm{D}_{3}$ molecules into LDP domains. This was confirmed by the surface compressional moduli. $\mathrm{D}_{3}$ interacted with lipid molecules in such manner that it increased monolayer rigidity $\left(\mathrm{K}_{\max }=134.8 \mathrm{mN} / \mathrm{m}\right)$, without changing the general organization of the LDP monolayer. The LDP- $\mathrm{D}_{3}$ mixed monolayer thus appeared more structured than the LDP one. $\mathrm{D}_{3}$ behavior resembles that of cholesterol in phospholipid monolayers, however without the condensing effect of the sterol (Ambike et al. 2011). The higher rigidity of LDP for vesicles with rigid bilayers (Kuntsche et al. 2011). domains, rather than the opposite. Indeed, the $\pi$-A isotherm profile of the LDP-RP monolayer is similar to that of RP alone. The insertion of lipid molecules into RP domains is also attested by the increase in the collapse surface pressure from 16 to $22 \mathrm{mN} / \mathrm{m}$. Partial collapse is confirmed by the decrease in the compressibility modulus above $22 \mathrm{mN} / \mathrm{m}$. Thus, RP led to a destructuration of the LDP mixed monolayer and when the surface density of the monolayer increased, the vitamin was partially squeezed out from the interface. The calculated $\Delta \mathrm{G}^{E X C}$ 
values for both systems suggest that insertion of $\mathrm{D}_{3}$ into LDP domains was controlled by favorable (attractive) interactions, whereas mixing of RP with LDP was limited due to unfavorable (repulsive) interactions, even at low surface pressures. According to Asai and Watanabe (2000), RP can be partially solubilized in the bilayer of phospholipids (up to 3 $\mathrm{mol} \%$ ), and the excess is separated from the phospholipids, and dispersed as emulsion droplets stabilized by a phospholipid monolayer.

On the whole, the information obtained regarding the interactions of the two vitamins with $\mathrm{NaTC}$ and LDP explain why $\mathrm{D}_{3}$ is more soluble than RP in an aqueous medium rich in mixed micelles. Both vitamins can insert into pure NaTC domains, but only $\mathrm{D}_{3}$ can also insert into the LDP domains in LDP-enriched NaTC micelles.

Furthermore, the results obtained suggest that this is not the only explanation. Indeed, since it has been suggested that $\mathrm{D}_{3}$ could form cylindrical micelle-like aggregates (Meredith et al. 1984), we hypothesize that the very high solubility of $\mathrm{D}_{3}$ in the aqueous medium rich in mixed micelles was partly due to the solubilization of a fraction of $\mathrm{D}_{3}$ as self-aggregates. Indeed, we observed that $D_{3}$ at concentrations higher than $0.45 \mu \mathrm{M}$, could self-assemble into various structures including nano-fibers. To our knowledge, no such structures, especially nanofibers, have been reported for $\mathrm{D}_{3}$ so far. Rod diameter was smaller than $10 \mathrm{~nm}$, much smaller than for the rods formed by lithocholic acid, for example (Terech et al. 2002). They were similar to those observed in highly concentrated LDP-NaTC mixtures, which seemed formed via desorganization of lipid vesicles. Disk-like and aggregates with unidentified structure, also observed in concentrated $\mathrm{D}_{3}$ samples, could be related to these nano-fibers.

In our solubilization experiments, which were performed at much higher $\mathrm{D}_{3}$ concentrations, both insertion of $\mathrm{D}_{3}$ molecules into NaTC and LDP domains, and $\mathrm{D}_{3}$ self- 
assembling could occur, depending on the kinetics of insertion of $\mathrm{D}_{3}$ into the NaTC-DLP mixed micelles.

\section{Conclusion}

The solubilization of a hydrophobic compound in bile salt-lipid micelles is dependent upon its chemical structure and its ability to interact with the mixed micelles components. Most hydrophobic compounds are expected to insert into the bile salt-lipid micelles. The extent of the solubilizing effect is, however, much more difficult to predict. As shown by others before us, mixed micelles components form a heterogeneous system with various molecular assemblies differing in shape and composition. The conditions of the medium $(\mathrm{pH}$, ionic strength and temperature) affect the formation of these molecular assemblies, although we did not study this effect on our system. Our results showed that $\mathrm{D}_{3}$ displayed a higher solubility in mixed micelle solutions than RP. This difference was attributed to the different abilities of the two vitamins to insert in between micelle components, but it was also explained by the propensity of $\mathrm{D}_{3}$, contrarily to $\mathrm{RP}$, to self-associate into structures that are readily soluble in the aqueous phase. It is difficult to predict the propensity of a compound to self-association. We propose here a methodology that was efficient to distinguish between two solubilizing behaviors, and could be easily used to predict the solubilization efficiency of other hydrophobic compounds. Whether the $\mathrm{D}_{3}$ self-assemblies are available for absorption by the intestinal cells needs further studies.

Acknowledgements: The authors are grateful to Dr Sylvain Trépout (Institut Curie, Orsay, France) for his contribution to cryoTEM experiments and the fruitful discussions. 
Funding: This study was funded by Adisseo France SAS.

489

490

Conflicts of interest: DP, ED and VLD are employed by Adisseo. Adisseo markets formulated

491

492

493

494

495

496

497

498

499

500

501

502

503

504

505

506

507

508

509

510

511

512

513

514

vitamins for animal nutrition.

\section{References}

Ambike, A., Rosilio, V., Stella, B., Lepetre-Mouelhi, S., \& Couvreur, P. (2011) Interaction of self-assembled squalenoyl gemcitabine nanoparticles with phospholipid-cholesterol monolayers mimicking a biomembrane. Langmuir, 27, 4891-4899.

Asai, Y., \& Watanabe, S. (2000) Formation and stability of the dispersed particles composed of retinyl palmitate and phosphatidylcholine. Pharmaceutical Development and Technology, 5, $39-45$.

Baskin, R., \& Frost, L. D. (2008) Bile salt-phospholipid aggregation at submicellar concentrations. Colloids and Surface B: Biointerfaces, 62, 238-242.

Bottari, E., D'Archivio, A. A., Festa, M. R., Galantini, L., \& Giglio, E. (1999) Structure and composition of sodium taurocholate micellar aggregates. Langmuir, 15, 2996-2998.

Borel, P., Caillaud, D., \& Cano, N. J. (2015) Vitamin D bioavailability: state of the art. Critical Reviews in Food Science and Nutrition, 55, 1193-1205.

Borel, P., \& Desmarchelier, C. (2017) Genetic Variations Associated with Vitamin A Status and Vitamin A Bioavailability. Nutrients, 9, 246. 
Cheng, C.-Y., Oh, H., Wang, T.-Y., Ragavan, S.R. \& Tung, S.-H. (2014) Mixtures of lecithin and bile salt can form highly viscous wormlike micellar solutions in water. Langmuir, 30, 10221-10230.

Cone, R.A. (2009) Barrier properties of mucus. Advanced drug delivery reviews, 61, 75-85.

Da Cunha, M. M., Trepout, S., Messaoudi, C., Wu, T. D., Ortega, R., Guerquin-Kern, J. L., \& Marco, S. (2016) Overview of chemical imaging methods to address biological questions. Micron, 84, 23-36.

Davies, J. T., \& Rideal, E. K. (1963) Interfacial phenomena 2nd ed. Academic press, p. 265.

Desmarchelier, C., Tourniaire, F., Preveraud, D. P., Samson-Kremser, C., Crenon, I., Rosilio, V., \& Borel, P. (2013) The distribution and relative hydrolysis of tocopheryl acetate in the different matrices coexisting in the lumen of the small intestine during digestion could explain its low bioavailability. Molecular Nutrition and Food Research, 57, 1237-1245.

Desmarchelier, C., Margier, M., Preveraud, D., Nowicki, M., Rosilio, V., Borel, P., \& Reboul, E. (2017) Comparison of the micellar incorporation and the uptake of cholecalciferol, 25hydroxycholecalciferol and 1- $\alpha$-hydroxycholecalciferol by the intestinal cell, Nutrients, $\underline{9}$, 1152.

Desmarchelier, C., \& Borel, P. (2017) Overview of carotenoid bioavailability determinants: From dietary factors to host genetic variations. Trends in Food Science \& Technology, 69, 270280.

Essaid, D., Rosilio, V., Daghildjian, K., Solgadi, A., Vergnaud, J., Kasselouri, A., \& Chaminade, P. (2016) Biochimica Biophysica Acta, 1858, 2725-2736. 
544 Fatouros, D.G., Walrand, I., Bergenstahl, B., Müllertz, A. (2009) Colloidal structures in media 545 simulating intestinal fed state conditions with and without lypolysis products. Pharmaceutical Research, 26, 361-374.

547

548

549

550

551

552

553

554

555

556

557

558

559

560

561

562

563

564

565

566

567

568

569

570

571

572

573

Flasinski, M., Wydro, P., \& Broniatowski, M. (2014) Lyso-phosphatidylcholines in Langmuir monolayers-influence of chain length on physicochemical characteristics of single-chained lipids. Journal of Colloid and Interface Science, 418, 20-30.

Gleize, B., Nowicki, M., Daval, C., Koutnikova, H., and Borel, P. (2016) Form of phytosterols and food matrix in which they are incorporated modulate their incorporation into mixed micelles and impact cholesterol micellarization. Molecular Nutrition and Food Research, 60, $749-759$

Heffner, C. T. R., Pocivavsek, L., Birukova, A. A., Moldobaeva, N., Bochkov, V. N., Lee, K. Y. C., \& Birukov, K. G. (2013) Thermodynamic and kinetic investigations of the release of oxidized phospholipids from lipid membranes and its effect on vascular integrity. Chemistry and Physics of lipids 175-176, 9-19.

Hernell, O., Staggers, J. E., \& Carey, M. C. (1990) Physical-chemical behavior of dietary and biliary lipids during intestinal digestion and absorption. 2. Phase analysis and aggregation states of luminal lipids during duodenal fat digestion in healthy adult human beings. Biochemistry, 29, 2041-2056.

Hollander, D., Muralidhara, K. S., \& Zimmerman, A. (1978) Vitamin D-3 intestinal absorption in vivo: influence of fatty acids, bile salts, and perfusate $\mathrm{pH}$ on absorption. Gut, 19, 267-272.

Huynh, L., Perrot, N., Beswick, V., Rosilio, V., Curmi, P. A., Sanson, A., \& Jamin, N. (2014) Structural properties of POPC monolayers under lateral compression: computer simulations analysis. Langmuir, 30, 564-573. 
574 Koo, S. I., \& Noh, S. K. (2001) Phosphatidylcholine inhibits and lysophosphatidylcholine 575 enhances the lymphatic absorption of alpha-tocopherol in adult rats. Journal of Nutrition, 131, $576 \quad 717-722$.

577

578 Kratohvil, J. P., Hsu, W. P., Jacobs, M. A., Aminabhavi, T. M., \& Mukunoki, Y. (1983) 579 Concentration-dependent aggregation patterns of conjugated bile-salts in aqueous sodium580 chloride solutions - a comparison between sodium taurodeoxycholate and sodium taurocholate. 581 Colloid and Polymer Science, 261, 781-785.

582

583

584

585

586

587

588

589

590

591

592

593

594

595

596

597

598

599

600

601

Kuntsche, J., Horst, J.C, \& Bunjes, H., Cryogenic transmission electron microscopy (cryoTEM) for studying the morphology of collidal drug delivery systems (2011) International Journal of Pharmaceutics 417, 120-137.

Leng, J., Egelhaaf, S.U., \& Cates, M.E. (2003) Kinetics of the micelle-to-vesicle transition ; aquous lecithin-bile salt mixtures, Biophysical Journal, 85, 1624-1646.

Madenci, D., \& Egelhaaf, S. U. (2010) Self-assembly in aqueous bile salt solutions. Current Opinion in Colloid and Interface Science, 15, 109-115.

Maislos, M., \& Shany, S. (1987) Bile salt deficiency and the absorption of vitamin D metabolites. In vivo study in the rat. Israel Journal of Medical Sciences, 23, 1114-1117

Meredith, S. C., Bolt, M. J., \& Rosenberg, I. H. (1984) The Supramolecular Structure of Vitamin-D3 in Water. Journal of Colloid and Interface Science, 99, 244-255.

Meyerhoffer, S. M., \& Mcgown, L. B. (1990) Critical Micelle Concentration Behavior of Sodium Taurocholate in Water. Langmuir, 6, 187-191. 
602

603

604

605

606

607

608

609

610

611

612

613

614

615

616

617

618

619

620

621

622

623

624

625

626

627

628

629

630

Pezron, I., Pezron, E., Claesson, P. M., \& Bergenstahl, B. A. (1991) Monoglyceride SurfaceFilms - Stability and Interlayer Interactions. Journal of Colloid and Interface Science, 144, 449457.

Rautureau, M., \& Rambaud, J. C. (1981) Aqueous solubilisation of vitamin D3 in normal man. Gut, 22, 393-397.

Reboul, E., Abou, L., Mikail, C., Ghiringhelli, O., Andre, M., Portugal, H., JourdheuilRahmani, D., Amiot, M. J., Lairon, D., \& Borel, P. (2005) Lutein transport by Caco-2 TC-7 cells occurs partly by a facilitated process involving the scavenger receptor class B type I (SRBI). Biochemical Journal, 387, 455-461.

Reboul, E., \& Borel, P. (2011) Proteins involved in uptake, intracellular transport and basolateral secretion of fat-soluble vitamins and carotenoids by mammalian enterocytes. Progress in Lipid Research, 50, 388-402.

Salentinig, S., Sagalowicz, L., \& Glatter, O. (2010) Self-assembled structures and pKa value of oleic acid in systems of biological relevance, Langmuir 26, 11670-11679.

Sy, C., Gleize, B., Dangles, O., Landrier, J. F., Veyrat, C. C., \& Borel, P. (2012) Effects of physicochemical properties of carotenoids on their bioaccessibility, intestinal cell uptake, and blood and tissue concentrations. Molecular Nutrition and Food Research, 56, 1385-1397.

Terech, P., de Geyer, A., Struth, B., \& Talmon, Y. (2002) Self-assembled monodisperse steroid nanotubes in water. Advanced Materials, 14, 495-498.

Tomoaia-Cotisel, M., Zsako, J., Mocanu, A., Lupea, M., \& Chifu, E. (1987) Insoluble mixed monolayers. Journal of Colloid and Interface Science, 117, 464-476. 
631 Tyssandier, V., Reboul, E., Dumas, J.-F., Bouteloup-Demange, C., Armand, M., Marcand, J., 632 Sallas, M., \& Borel, P. (2003) Processing of vegetable-borne carotenoids in the human stomach 633 and duodenum. American Journal of Physiology - Gastrointestinal and Liver Physiology, 284, 634 G913-G923.

635

636 Walter, A., Vinson, P.K., Kaplun, A., \& Talmon, Y. (1991) Intermediate structures in the 637 cholate-phosphatidylcholine vesicle-micelle transition, Biophysical Journal, 60, 1315-1325.

638

639 Yang, Y., \& McClements, D. J. (2013) Vitamin E and vitamin E acetate solubilization in mixed 640 micelles: physicochemical basis of bioaccessibility. Journal of Colloid and Interface Science, $641405,312-321$.

642

643 
646

647

648

649

650

651

652

653

654

655

656

657

658

659

660

661

662

663

664

665

666

667

\section{Figure Captions}

Figure 1: Chemical structures for $\mathrm{D}_{3}$ and RP.

Figure 2: Solubilization of $\mathrm{D}_{3}$ and RP in aqueous solutions rich in mixed micelles: cholecalciferol; $(\square)$, retinyl palmitate. For $\mathrm{D}_{3} \mathrm{R}^{2}=0.98$, and regression slope $=0.71$.

Figure 3: Cryo-TEM morphology of (A) $15 \mathrm{mM}$ mixed LDP-NaTC micelles, (B) and (C) 5 mM mixed LDP-NaTC micelles $+1.7 \mathrm{mM} \mathrm{D}_{3}$, (D) $15 \mathrm{mM}$ mixed LDP-NaTC micelles $+5 \mathrm{mM}$ $\mathrm{D}_{3}$, (E) and (F) pure $\mathrm{D}_{3}$ assemblies: $(a)$ spherical vesicles, $\left(a^{\prime}\right)$ small deformed vesicles, $(b)$ nano-fibers, $(c)$ aggregates, $(d)$ large deformed vesicle fragments (sheets), (e) disk-like nanoassembly, $(f)$ emulsion-like droplet, $(g)$ large deformed vesicle, $(h)$ disk-like nano-assemblies, (i) nano-fibers, $(h)$ aggregates. All samples were prepared by the dry film hydration method, without filtration. Scale bars: $100 \mathrm{~nm}$.

Figure 4: Mean compression isotherms for (A) the pure micelles components and the LDP mixture, and (B) $\mathrm{D}_{3}$ (solid line) and RP (dashed line) spread at the air/buffer interface from a chloroform-methanol $(9: 1)$ solution $\left(25^{\circ} \mathrm{C}\right) . \operatorname{POPC}(\bullet), \operatorname{LysoPC}(\mathrm{O})$, monoolein $(\triangle)$, cholesterol ( $\mathbf{\Delta}$ ), oleic acid ( $\square)$, LDP (dashed line).

Figure 5: (A) Adsorption isotherms for LDP hydrated in NaTC-free buffer (O), LDP hydrated in NaTC-containing buffer solution ( $\square$ ), and pure NaTC ( $\bullet$ solutions at $25^{\circ} \mathrm{C}$. (B) Surface pressure increment upon injection of NaTC beneath a condensed LDP monolayer $\left(\pi_{\mathrm{i}}=28\right.$ $\mathrm{mN} / \mathrm{m}$ ). The final NaTC concentration in the subphase was $0.1 \mathrm{mM}$. The initial increase in surface pressure following NaTC addition to the subphase was an injection effect. 
669 Figure 6: $\pi-A$ isotherms (A,B), compressibility moduli (C) and excess free energies (D) for 670 LDP-vitamin monolayers $\left(25^{\circ} \mathrm{C}\right)$. A: LDP mixture (dashed line), pure $\mathrm{D}_{3}$ (dashed-dot line) and 671 LDP-D $_{3}$ (7:3) mixture (solid line); B: LDP mixture (dashed line), RP (dashed-dot line) and 672 LDP-RP (7:3) mixture (solid line). C: LDP mixture (dashed line), LDP-D 3 (7:3) mixture (solid 673 line), and LDP-RP (7:3) mixture (dashed-dot-dot line). D: LDP-D 3 (7:3) (square) and LDP-RP $674 \quad$ (7:3) (triangle) mixtures.

Figure 1

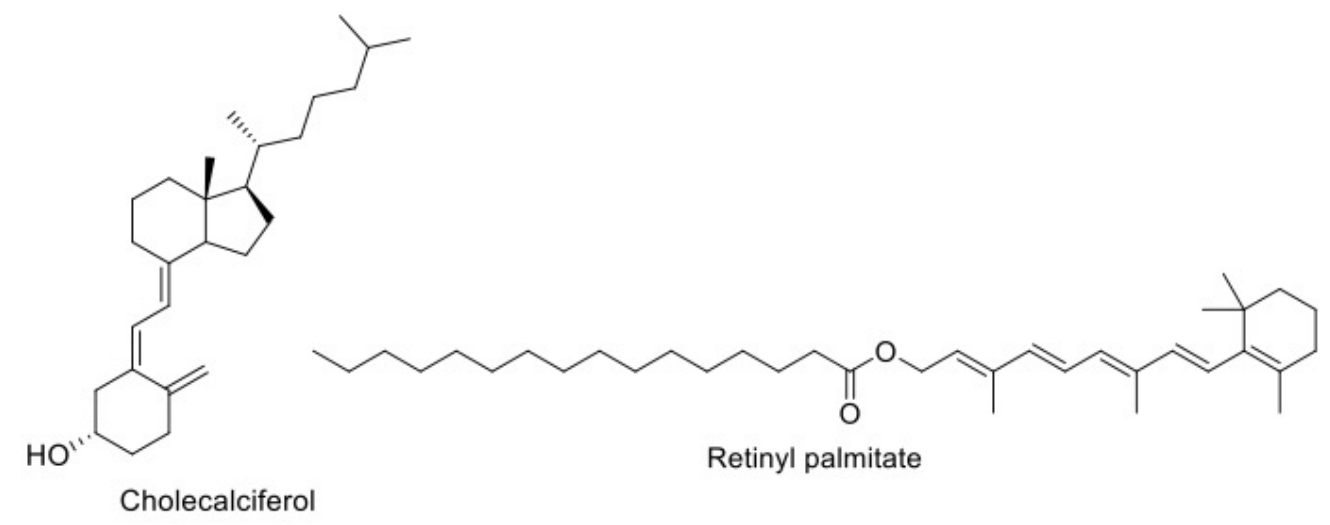

Figure 2

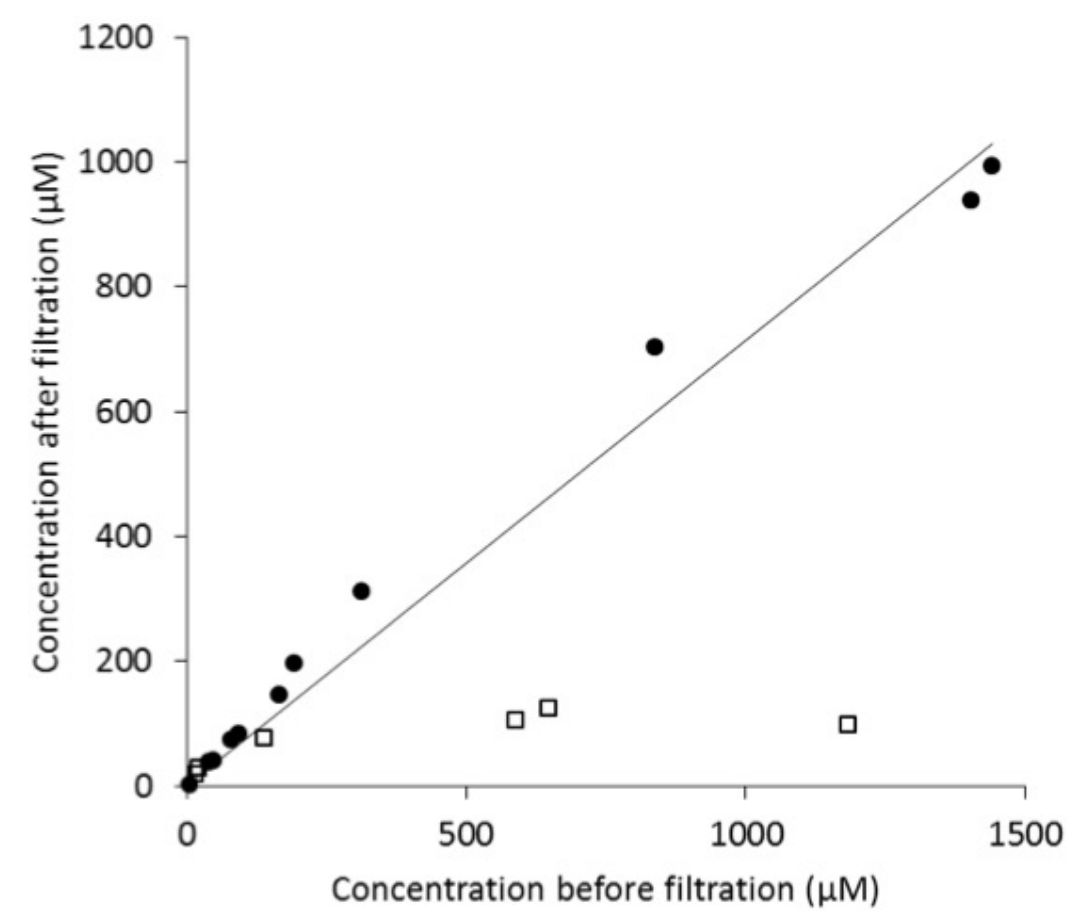


Figure 3
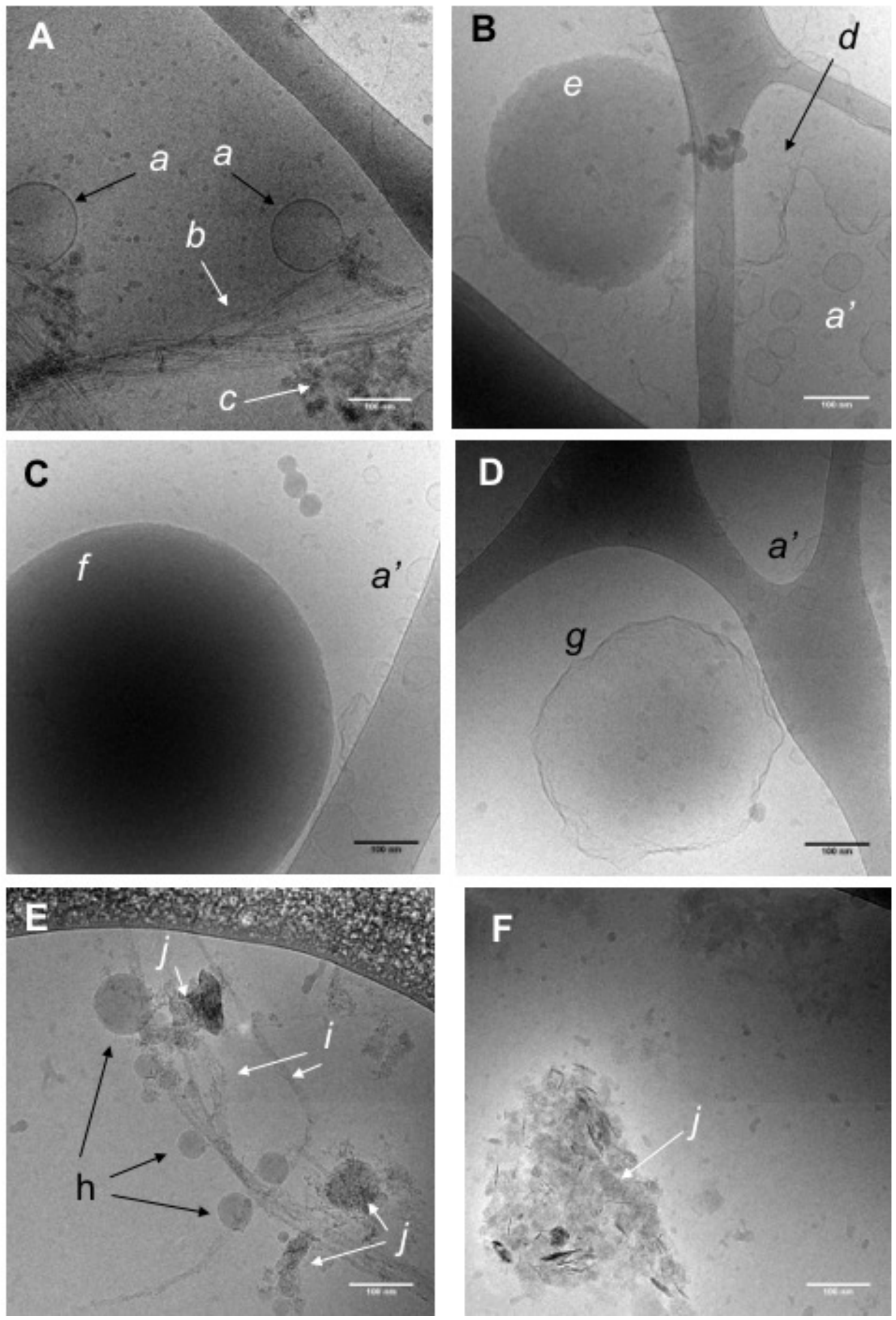

F 
Figure 4
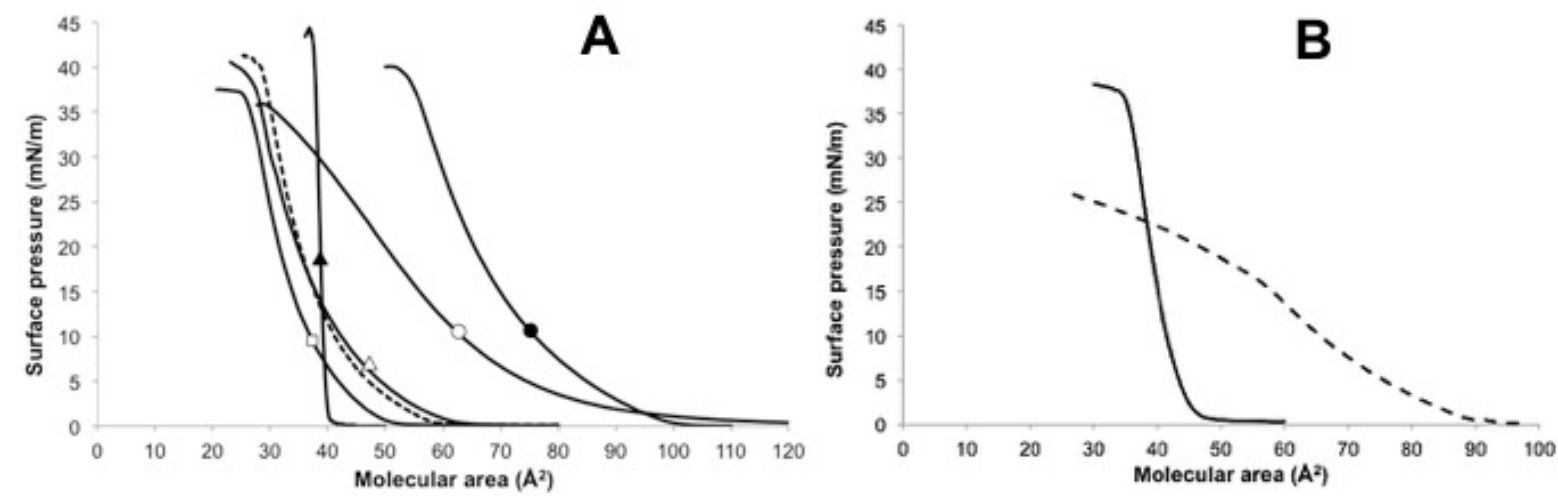

Figure 5
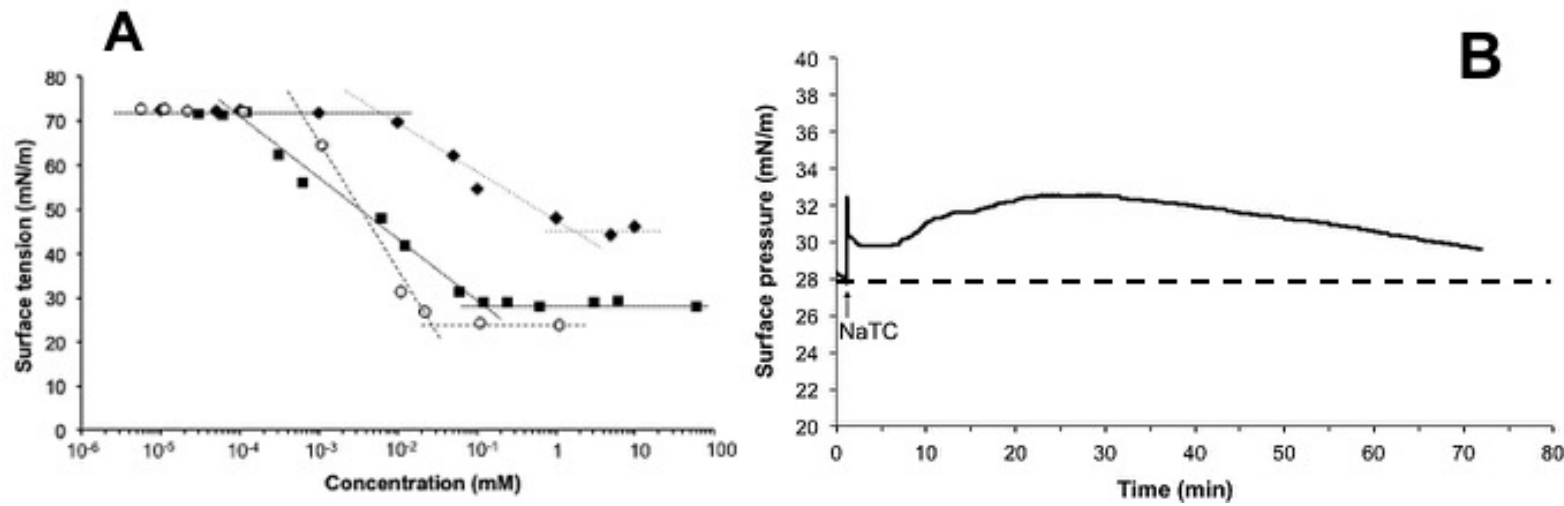
Figure 6
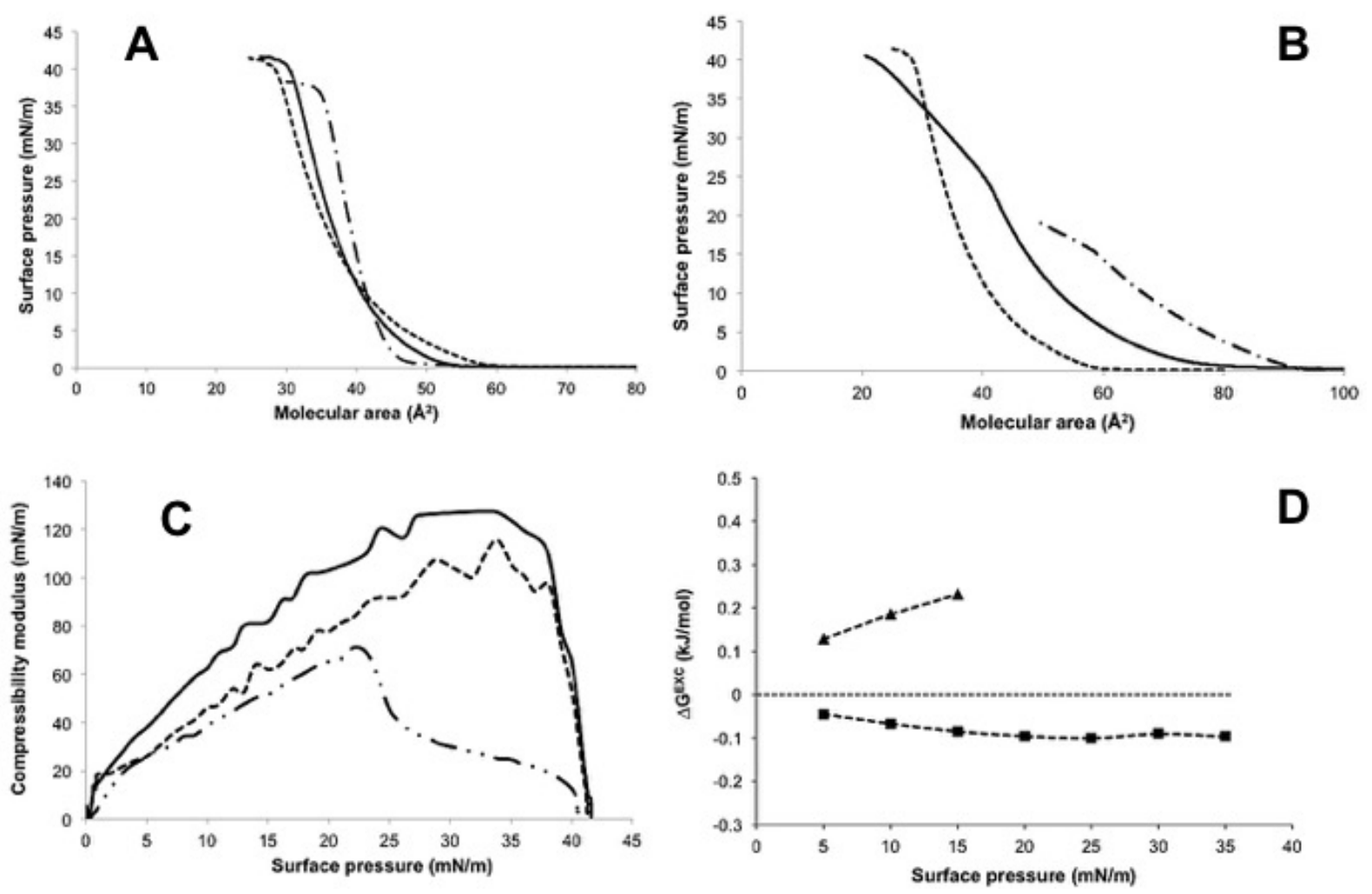

Highlights :

Cholecalciferol $\mathrm{D}_{3}$ exhibits dramatic solubilization in simulated intestinal medium

Both lipids and bile salt in mixed micelles contribute to $\mathrm{D}_{3}$ solubilization

$\mathrm{D}_{3}$ self-association is an additional factor impacting the solubilization process

Retinyl palmitate (RP) is poorly solubilized in the mixed micelle solutions

High excess free energy of mixing of RP with the lipids explains its poor inclusion 